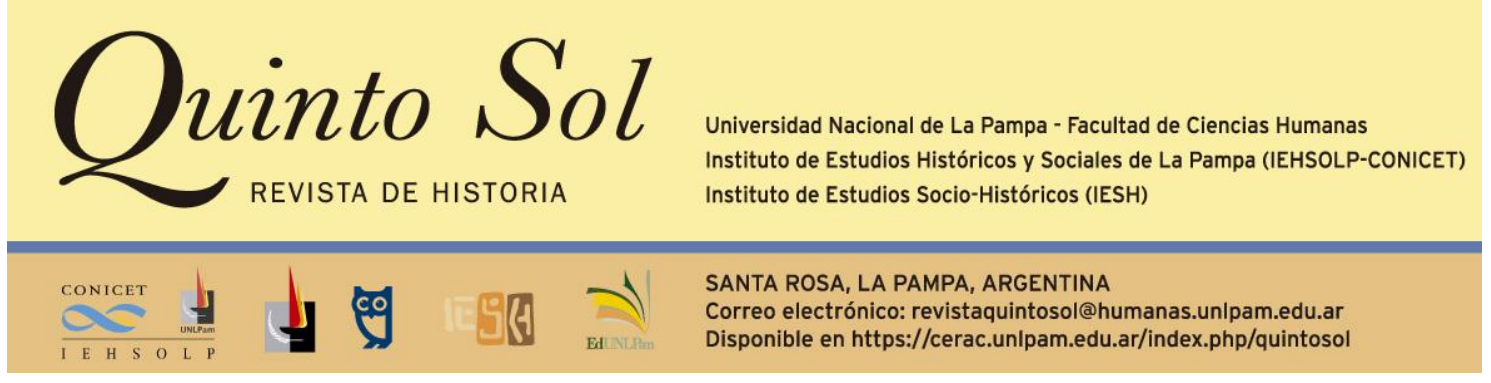

Quinto Sol, vol. 26, n 1, enero-abril 2022, ISSN 1851-2879, pp. 1-21

http://dx.doi.org/10.19137/qs.v26i1.5783

Esta obra se publica bajo licencia Creative Commons 4.0 Internacional. (Atribución-No ComercialCompartir Igual)

\title{
Estado y salud pública en la provincia de Buenos Aires: proyectos y prácticas durante el peronismo clásico
}

\author{
State and Public Health in Buenos Aires Province: Classic \\ Peronism's Projects and Practices
}

\section{O Estado e a saúde pública na província de Buenos Aires: projetos e práticas durante o peronismo clássico}

\section{María Liliana Da Orden}

Consejo Nacional de Investigaciones Científicas y Técnicas/Universidad Nacional de Mar del Plata Instituto de Humanidades y Ciencias Sociales. Centro de Estudios Históricos

Argentina

Correo electrónico: mldaor9@gmail.com

\section{Resumen}

El artículo analiza la política de salud de la provincia de Buenos Aires durante los gobiernos peronistas de Domingo Mercante y Carlos Aloé (1946-1955). En primer lugar, se considera el tratamiento dado por el oficialismo y la oposición a la creación del Ministerio de Salud Pública y Asistencia Social, y a los planes de obras públicas vinculados con este. En la segunda parte se hace foco en las prácticas de gobierno teniendo en cuenta el perfil de los funcionarios del ministerio, la expansión efectiva de los establecimientos de salud y los recursos financieros y personales

Palabras clave política de salud peronismo Estado 
Da Orden, M.L. Estado y salud pública en la provincia de Buenos Aires: proyectos y prácticas durante el peronismo clásico

asignados. Los resultados sugieren que la política sanitaria adquirió una relevancia que no había tenido hasta entonces, aunque algunos condicionamientos coyunturales y otros de más largo plazo limitaron los alcances del cambio. Asimismo, tanto las disposiciones legales como las prácticas referidas a las instituciones tradicionales evidencian que el Estado provincial no pretendía superar la fragmentación existente.

\section{Abstract}

This article analyzes the public health policy of Peronist governors (19461955) in Buenos Aires Province. I consider both the government and the opposition treatment to the Ministry of Public Health creation and the construction plans that formed the public health policy. In the second part I focus on the practices related to public management, and the trajectories of ministry officials. Financial and personal resources assigned and the effective expansion of the health services are analyzed. The results suggest that provincial health policy had a significant development but short-term and long-term constraints limited the changes. In addition, legal provisions such as practices referring to traditional health institutions show that the provincial State was conceived as a coordinating entity of the health system rather than a unifying one.

\section{Resumo}

O trabalho analisa a política de saúde da Província de Buenos Aires durante os governos peronistas de Domingo Mercante e Carlos Aloé (1946-1955). Em primeiro lugar, considera-se o tratamento outorgado pelo oficialismo pró-governo à criação do Ministério de Saúde Pública e Assistência Social e aos planos de obras públicas relacionadas a este. Numa segunda parte, focaliza-se nas práticas de governo levando em consideração o perfil dos funcionários do ministério, a expansão efetiva dos estabelecimentos de saúde e os recursos financeiros e pessoais assignados. Os resultados sugerem que a política sanitária adquiriu uma relevância que não tinha tido até esse momento a pesar de que alguns condicionamentos conjunturais e outros de longo prazo limitaram os alcances da mudança. Porém, tanto as disposições legais quanto as práticas referidas às instituições tradicionais evidenciam que o Estado provincial não pretendia superar a fragmentação existente.

Palavras-chave políticas de saúde peronismo Estado

Recepción del original: 24 de abril de 2021.

Aceptado para publicar: 23 de septiembre de 2021. 


\section{Estado y salud pública en la provincia de Buenos Aires: proyectos y prácticas durante el peronismo clásico}

Desde la reforma del aparato estatal a las prácticas sanitarias, el gobierno de Juan Domingo Perón tuvo y/o esgrimió uno de sus logros más visibles en el ámbito de la salud. Es cierto que tal vez fue en esa dimensión donde también la "idiosincrasia" del peronismo se hizo más patente. De hecho, la mayor crítica que se le formula es la persistencia y aun el refuerzo de la fragmentación vigente frente a la deseable unificación del sistema. A la tensión existente entre el Ministerio de Salud Pública de la nación y la Fundación Eva Perón (FEP), se habría añadido la acción de los sindicatos con sus mutuales u obras sociales. De manera que, como consecuencia de la politización a la cual estuvo sometido el sistema, el peronismo habría continuado y acentuado los problemas que llevarían a la crisis. Esta falta de unificación ha sido vista además como el principio de una serie de medidas que imposibilitaron la universalización de la salud pública en Argentina (Belmartino, 2005; Ross, 2007; Ramaciotti, 2009).

Sin embargo, a pesar de la difusión que tuviera la acción nacional en el área, no era esta la esfera exclusiva de actuación. De acuerdo con el sistema federal de gobierno, eran las provincias, antes que el poder central, las que tenían jurisdicción directa sobre la salud pública de sus distritos. Estudios como los realizados para Mendoza, Córdoba o Santa Fe han puesto de relieve el peso decisivo de sus gobiernos tanto frente a los actores tradicionales como a un Estado nacional mucho menos presente de lo que la planificación y la propaganda hacían suponer (Rodríguez, 2013; Bacolla, 2016; Hirschegger, 2016; Ortiz Bergia, 2016).

De este modo, la salud pública en tiempos del peronismo se perfila como una realidad mucho más compleja que la que ofrece una visión del y desde el Estado nacional. La política sanitaria de la provincia de Buenos Aires - por su riqueza, pero también por sus variedades regionales- proporciona un ángulo particular para el análisis. Su zona industrializada, tan próxima a la sede del poder central y a las bases políticas del peronismo, sin duda propiciaba la intervención de la nación en el área. En ese contexto, ¿cuál fue el papel del gobierno provincial? ¿Cómo se organizó la salud? Y tal vez lo más importante, ¿cómo fue su funcionamiento?

Atendiendo no solo al diseño sino al quehacer cotidiano, la respuesta a algunas de estas cuestiones busca poner el acento en la complejidad que supone la salud tanto desde el punto de vista de la administración -con un ministerio que debía ponerse en marcha-, como de la relación con las entidades sociales que estaban en juego e incidían, a su vez, en la configuración estatal. Ambas dimensiones se hallan en la línea de los estudios que en los últimos años ponen en evidencia la heterogeneidad y porosidad que caracteriza al Estado visto desde "adentro" (Bohoslavsky y Soprano, 2010).

Aunque las fuentes oficiales que utilizo favorecen el estudio de la dimensión prescriptiva, un análisis detallado y no lineal habilita la exploración de las prácticas y los 
Da Orden, M.L. Estado y salud pública en la provincia de Buenos Aires: proyectos y prácticas durante el peronismo clásico

intereses en pugna. En el marco de un gobierno que pretendía transformar al mismo tiempo todas o casi todas las esferas de acción estatal, a la vez que estaba inmerso en la organización de la nueva fuerza partidaria, las políticas de salud no solo acusaron las tensiones que de ello se derivaban sino también los condicionamientos existentes, desde los recursos y el personal disponible hasta la actuación de las organizaciones sociales tradicionales. Con esta proposición busco aportar una visión un poco más realista -histórica, podríamos decir- que la de aquellas que parten de la premisa de un sistema deseable de salud, tratando de iluminar desde esta perspectiva el papel del gobierno nacional en el área.

\section{La salud pública bonaerense: realidades y medidas de cambio}

Antes de avanzar en el tema, veamos algunas cifras que permiten dimensionar la escala del distrito que nos ocupa, una cuestión no menor para nuestro análisis. Promediando la década del cuarenta residían en la provincia de Buenos Aires algo más de cuatro millones de habitantes, la cuarta parte del total de la población. Esta se hallaba distribuida de modo desparejo en un territorio de unos 307.000 kilómetros cuadrados -una extensión que casi duplica la de la provincia de Córdoba, la más extensa por entonces-. Siete de sus concentraciones urbanas rondaban los 100.000 habitantes, esto es, una población similar a la de distritos del noroeste como La Rioja, Catamarca o Jujuy, en tanto que cinco ciudades contaban con una población cercana al cuarto de millón, cifra análoga a la de provincias como Salta o San Juan. Existían además fuertes contrastes internos: cerca de un millón de habitantes estaban nucleados en el Gran Buenos Aires, crecientemente industrializado. Con menor población se hallaban las ciudades de La Plata, capital de la provincia, Mar del Plata y Bahía Blanca que, junto con algunos núcleos intermedios, sobresalían en el vasto espacio rural. ${ }^{1}$ De manera que, si se trataba de la provincia más rica, los contrastes físicos y demográficos no solo eran patentes frente al resto de los distritos sino también al interior bonaerense.

En ese contexto, ¿qué papel desempeñaba el gobierno provincial en el ámbito de la salud? Hasta bien entrado el siglo XX su presencia apuntaba a la "higiene defensiva" (Armus y Belmartino, 2001), es decir, al sostenimiento de hospitales destinados a enfermedades mentales e infecto-contagiosas. Esto insumía cerca del $70 \%$ de las 4801 camas que estaban bajo su órbita. La atención prestada a la medicina general era, pues, secundaria y estaba concentrada en la ciudad capital y algunas otras del interior. Era el caso de los hospitales regionales del norte y noroeste provincial (General Villegas, Junín, Lobos y Zárate) y de los policlínicos de Bahía Blanca y Haedo. Salvo este último, que fue inaugurado en el conurbano a principios de los años cuarenta, casi todos tenían varias décadas de funcionamiento. En conjunto, se trataba de 15 hospitales gestionados por la Dirección General de Higiene (DGH), dependiente del Ministerio de Obras Públicas, un hecho de por sí ilustrativo. Por su parte, la nación

\footnotetext{
${ }^{1}$ Ver al respecto IV Censo General de la Nación, (1951, Tomo 1). En Referencias bibliográficas se consignan los datos completos de fuentes éditas.
} 
Da Orden, M.L. Estado y salud pública en la provincia de Buenos Aires: proyectos y prácticas durante el peronismo clásico

sostenía cinco instituciones destinadas a enfermos contagiosos o mentales procedentes de distintos puntos del país. ${ }^{2}$

El grueso de la atención sanitaria quedaba así en manos de entidades públicas o particulares sin fines de lucro de distinta envergadura. Asociaciones de beneficencia, religiosas o simplemente civiles gestionaban medio centenar de hospitales (el $27 \%$ del total de camas). Llamativamente, el mayor peso recaía en los municipios, que sostenían 68 establecimientos con cerca del $40 \%$ de la capacidad de internación. Una situación que no se condecía con los limitados recursos que tenían bajo su órbita. Este papel iba de la mano del carácter subsidiario que se asignaba al Estado provincial en el área de salud. Así, una pequeña porción de los recursos de las distintas instituciones provenía de las arcas provinciales, ${ }^{3}$ que también en parte solventaba la ampliación o refacción de las instalaciones. De manera que esta situación precaria debía compensarse con colectas y donaciones.

Más allá de la medicina tradicional o de la atención en sanatorios privados, la salud se hallaba, pues, afectada por las limitaciones que suponía la fragmentación en organismos de distinto alcance y el déficit de recursos. Esto sin considerar las carencias de las zonas rurales, distantes de los centros sanitarios. ${ }^{4}$

En los años treinta o aun antes, esta situación -que no era exclusiva de la provincia- había suscitado críticas y debates que llamaron la atención sobre la existencia de una "cuestión hospitalaria" (Belmartino, Block, Carnino y Persello, 1991). Como en otras dimensiones de la vida pública, la injerencia estatal fue considerada una salida a la crisis que aquejaba al conjunto del sistema sanitario. Si en 1941 la experiencia más notable se dio en la provincia de Santa Fe, con la creación de un ministerio, la Secretaría de Salud Pública de la Nación -instaurada poco antes de la llegada de Perón a la presidencia- iba a seguir ese derrotero (Veronelli y Veronelli Correch, 2004). No es extraño que, en 1946, apenas llegado a la gobernación, Domingo Mercante enviara a la Cámara de Diputados un proyecto sobre el tema. A este se sumaron las iniciativas de dos diputados oficialistas de distintas líneas políticas: Juan C. Zamudio, radical de la Junta Renovadora, y Alfredo Galeano, laborista. Los tres proyectos buscaban jerarquizar el rol del poder provincial y reemplazar el tradicional concepto de higiene por el más amplio de salud pública. Sin embargo, solo el laborista proponía la creación de un ministerio, un indicio que, como se verá, da cuenta del carácter experimental de la política del Poder Ejecutivo, que rápidamente modificó su propuesta. Más allá del cuestionamiento de un senador del radicalismo opositor, que proponía la creación de una entidad autárquica "con su economía lejos de la Casa de

\footnotetext{
${ }^{2}$ La tercera parte de los 14.344 lechos disponibles en el distrito correspondían a nosocomios provinciales. Estos y los siguientes datos provienen de: "Catastro Hospitalario" en Plan General de Trabajos Públicos, (1947, Volumen 1); Anuario Geográfico Argentino (AGA), (1941, p. 567).

${ }^{3}$ Desde principios del siglo XX el presupuesto provincial les asignaba un monto fijo por cama y la nación hacía lo propio. En la práctica, la demora de estas partidas no resolvía la penuria financiera que padecían estas instituciones. Ver, por ejemplo, el informe del diputado oficialista Roberto Cursack en Diario de Sesiones de la Cámara de Diputados (DSCD), (1946-47, Tomo I, pp. 2715-17).

${ }^{4}$ En otras provincias, el servicio hospitalario estaba en su mayor parte a cargo de instituciones de beneficencia o de caridad (más de dos tercios de los nosocomios). AGA, (1941, pp. 567-68, 571).
} 
Da Orden, M.L. Estado y salud pública en la provincia de Buenos Aires: proyectos y prácticas durante el peronismo clásico

Gobierno", ${ }^{5}$ lo cierto es que, con algunos matices, en ambas Cámaras hubo coincidencia sobre la necesidad de crear una agencia bajo la denominación Ministerio de Salud Pública y Asistencia Social, que dirigiera y coordinara el área.

En efecto, la Ley 5116 establecía que el nuevo ministerio debía "coordinar, unificar y racionalizar los servicios de salud e higiene existentes en el distrito" y facultaba al gobierno a realizar convenios con todas las instituciones, desde los municipios a las sociedades de beneficencia. ${ }^{6}$ Esa también había sido la tesitura de la oposición radical:

La creación de un ministerio de Salud Pública en la provincia más que un organismo estatal de centralización debe ser un organismo coordinador de los servicios sanitarios... no debe impedir que en la Provincia se sigan desarrollando todas las instituciones de carácter particular o municipal que tiendan al mejoramiento de la salud pública. ${ }^{7}$

Independientemente de la oposición a la concentración de poder con la que parecía amenazar el peronismo, la afirmación pone de manifiesto el apoyo que brindaban todos los sectores políticos a la articulación del poder provincial con las instituciones sociales existentes. De este modo, la anunciada unidad coexistía con la fragmentación del sistema.

Sí, en cambio, se establecía la unidad de comando de la asistencia social y la salud pública, una idea que, como tal, respondía a criterios ya concebidos en los años treinta (Ramaciotti, 2009; Biernat, 2016). En esta área, la principal novedad se refería a la maternidad y la niñez, únicas funciones que contaron con una nueva repartición. En efecto, en la práctica, el ministerio unificaba reparticiones ya existentes en dos de los tres departamentos que componían el gabinete: DGH, Consejo de Lucha Antituberculosa, Departamento Químico y Medicina Veterinaria (de Obras Públicas) y Minoridad, Educación Física y Cuerpo Médico de Cárceles (procedentes de Gobierno). El Cuerpo Médico Escolar provenía de la autárquica Dirección General de Escuelas. Se trataba pues de un conglomerado de agencias, organizadas en direcciones y divisiones, que pasaban a integrar las dos subsecretarías del nuevo ministerio. ${ }^{8}$

A pesar de las detalladas funciones y reparticiones, ya antes de la sanción se concebía que las disposiciones de esta ley cambiarían sobre la marcha. Tal como indicara un diputado oficialista, "Primero hay que crear el Ministerio de Salud Pública y a medida que se vayan viendo las necesidades, se harán las modificaciones

\footnotetext{
${ }^{5}$ Se trataba del médico Adolfo Bollini, ver Diario de Sesiones de la Cámara de Senadores (DSCS), (1946-47, Tomo III, p. 280).

${ }^{6}$ Artículo $1^{\circ}$ de la Ley 5116. https://normas.gba.gob.ar/ar-b/ley/1947/5116/10111. Todas las leyes citadas en adelante han sido consultadas en https://normas.gba.gob.ar

${ }^{7}$ Intervención del diputado Manuel Faya. En el mismo sentido se había expresado en el Senado el radical Ángel Galcerán. DSCD, (1946-47, Tomo V, p. 396); DSCS (1946-47, Tomo III, p. 3806).

${ }^{8}$ Las cuatro primeras estarían bajo la órbita de la Subsecretaría de Salud Pública, en tanto que las restantes compondrían a la Subsecretaría de Asistencia Social junto con la sección de Maternidad e Infancia, de nueva creación.
} 
Da Orden, M.L. Estado y salud pública en la provincia de Buenos Aires: proyectos y prácticas durante el peronismo clásico

necesarias". ${ }^{9}$ Así, las transformaciones serían diversas (en 1949, por ejemplo, las direcciones generales pasaron de cuatro a siete y otro tanto ocurrió con las direcciones).

El poder provincial había avanzado, pues, en el terreno de la salud con la urgencia de promover cambios simultáneos en casi todas las áreas de gobierno. ${ }^{10}$ En el mismo sentido habían procedido la mayoría de las provincias. ${ }^{11} \mathrm{Si}$ este tipo de transformaciones ha sido cuestionado como una imposición del gobierno nacional (Bacolla, 2016), lo cierto es que la creación de ministerios del área se apoyaba en ideas y demandas de larga data (Veronelli y Veronelli Correch, 2004) que ahora se concretaban. De manera que, tanto en su concepción como en su organización, al menos en esta provincia, la medida suponía un cambio significativo, pero también importantes continuidades.

Ahora bien, esta jerarquización de la salud debía tener un correlato en los centros sanitarios de gestión provincial, tan escasos y poco diversificados. En efecto, como primera medida fue aprobado un Plan Inicial de Obras Públicas que en parte retomaba iniciativas anteriores y anunciaba la voluntad de intervenir decididamente en diversas áreas. Con destino a la construcción o modificación de hospitales, unidades sanitarias y salas de primeros auxilios se reservaron 10 millones de pesos, el $15 \%$ del monto total. Esta disposición anticipaba la ambiciosa política del Plan General de Trabajos Públicos para el trienio 1947-49, ${ }^{12}$ que traducía, como en otras provincias, la disposición planificadora del poder central. Con una emisión de títulos de deuda interna de 863 millones de pesos -monto 3,7 veces superior al presupuesto provincial del primer año indicado- ${ }^{13}$ el espectro de construcciones abarcaba desde infraestructura hídrica y vial hasta hotelería y vivienda "obrera". Aquí las obras para atención de la salud representaban alrededor del 7\% del total. En su mayoría se trataba de edificaciones pequeñas y medianas con las que se esperaba triplicar el número de camas a cargo de la provincia. ${ }^{14}$ En 1949, a estas se sumaron otros 20 millones para un plan en gran parte destinado a ampliar los hospitales de La Plata y Haedo. Asimismo, dada su importancia como destino turístico, los partidos de General Pueyrredon y

\footnotetext{
${ }^{9}$ Se trataba del diputado Raúl Peláez, miembro informante de la Comisión de Higiene, DSCD, (1946-47, Tomo V p. 3972).

${ }^{10}$ En 1948, la Ley 5324 de organización de ministerios creó el de Gobernación, poco después suprimido. En 1949, la reforma de la constitución provincial completó el gabinete con los ministerios de Educación y Asuntos Agrarios.

${ }^{11}$ En 1952, nueve de las catorce provincias habían creado el Ministerio de Salud. La excepción la constituían Catamarca, La Rioja, San Juan y Santiago del Estero. Jujuy incorporó la denominación en uno de sus dos departamentos. Anuario Argentina, (1953, p. 201).

12 Plan Inicial de Trabajos Públicos (Ley 5079 de 1946) y Plan General de Trabajos Públicos, (1947, Volumen 1, Ley 5142. La planificación tenía antecedentes en el gobierno de Manuel Fresco, en el Plan de Trabajos Públicos para el trienio 1937-1938-1939, Ley 4539.

${ }^{13}$ Cálculo de Recursos y Presupuesto General de Gastos de la Administración y Reparticiones Autárquicas para el año 1947. DSCD, (1946-47, Tomo V, p. 4079).

${ }^{14}$ Se hallaban presupuestadas 146 obras (salas de primeros auxilios, centros de higiene y unidades sanitarias en su mayoría), así como ampliaciones o refacciones de hospitales municipales o privados con fines sociales. DSCD, (1946-47, Tomo I, pp. 1112-1114); Ministerio de Salud Pública y Asistencia Social (MSPyAS), (1947-1951, p. 21).
} 
Da Orden, M.L. Estado y salud pública en la provincia de Buenos Aires: proyectos y prácticas durante el peronismo clásico

Necochea fueron objeto de sendos planes que incluían salas de primeros auxilios y unidades sanitarias por más de 4 millones de pesos. ${ }^{15}$

Las modificaciones continuaron, bien a propuesta del Poder Ejecutivo o por iniciativa de los legisladores que buscaban atender los reclamos de sus distritos. De este modo, entre 1947 y 1949 se sumaron otras cincuenta obras. En conjunto fueron aprobadas así más de dos centenares de construcciones, ampliaciones o refacciones en todo el territorio provincial, donde se incluían -y esto es importante por la continuidad que supone- instituciones municipales y sociales ya existentes. ${ }^{16} \mathrm{El}$ total asignado en los tres primeros años de gobierno superó los 109 millones de pesos. Claro que la inflación creciente fue degradando esos valores, lo que dio origen, como veremos, a nuevas disposiciones. El hecho es que, también aquí, el diseño se fue modificando casi al tiempo de ser aprobado, una característica que, a pesar de la importancia concedida a la planificación, asimismo es observable tanto en el Primer Plan Quinquenal como en el Plan de Construcciones Sanitarias del gobierno nacional (De la Vega, 2017).

\section{Una aproximación a las prácticas}

Desde el punto de vista normativo, el gobierno provincial buscó ampliar su injerencia en el ámbito de la salud de manera decisiva. La puesta en marcha iba a implicar importantes desafíos, tanto desde el punto de vista de la concreción de las obras planificadas como de la actividad del nuevo ministerio. Como se verá, a esto se sumaba el hecho de que buena parte de los funcionarios encargados de la gestión a su vez estaban involucrados en una tarea por cierto ardua como fue la organización de la nueva fuerza partidaria.

Ahora bien, el funcionamiento de la repartición creada a partir de un conglomerado de agencias preexistentes no debe darse por sentado. Secciones que habían estado bajo la órbita de diferentes ministerios consolidados pasaban a formar parte de uno nuevo, mientras que otras debían crearse casi por completo -el área de maternidad e infancia, por ejemplo-. ¿Quiénes fueron los funcionarios que estuvieron a cargo?

Como ha señalado Oscar Aelo (2012), el ministerio fue conducido por técnicos, todos médicos de profesión. Sin embargo, esta caracterización resulta simplificadora si se tiene en cuenta que desde hacía décadas los médicos (junto con los abogados) nutrían los elencos de dirigentes de todos los partidos políticos. Veamos la cuestión con algo de detalle. Al momento de crearse, el núcleo de la nueva cartera estaba dirigido por Héctor Mercante, desde 1943 director de la Casa Cuna y del Policlínico de La Plata sucesivamente. Poco antes de las elecciones se había hecho cargo de la DGH,

\footnotetext{
${ }^{15}$ Plan de Obras de Salud Pública y Asistencia Social (Ley 5523 de 1949). En el mismo año se aprobaron los planes generales de los partidos citados (Leyes 5506 y 5520).

${ }^{16}$ Los principales beneficiarios fueron los hospitales municipales (Vicente López, Chacabuco, Saladillo, Rivadavia, General Lamadrid y Ramallo). También se incluyeron edificios sostenidos por entidades vecinales e instituciones de interés público (Liga Popular contra la Tuberculosis, Cruz Roja Argentina) o beneficencia. Las iniciativas provinieron tanto del oficialismo como del radicalismo. El monto total asignado rondó los 14 millones de pesos. Para 1947, ver Leyes 5146, 5151, 5217, 5219, 5224, 5227. En el año 1948, las Leyes 5372, 5373, 5394, 5422; y en 1949 las Leyes 5465, 5479, 5512, 5516, 5526.
} 
donde continuaría por más de un año. Este sería, pues, un paso de su carrera política que continuó en el estratégico Ministerio de Gobierno. ${ }^{17}$ Aunque, por un lado, esto formaba parte de las marchas y contramarchas de los primeros tiempos de la gestión de Mercante, también evidencia la inestabilidad de los funcionarios en la DGH. En efecto, desde 1943 se habían sucedido siete directores, una situación que venía dándose desde que una intervención nacional desplazara al gobernador Manuel Fresco en 1940. Hasta ese momento, la DGH había estado a cargo de Atilio Viale, un médico de trayectoria conservadora (comisionado de Saladillo, su municipio de origen, en dos oportunidades), que había dirigido el Ministerio de Obras Públicas y tras una derrota partidaria pasó a la dirección que nos ocupa. ${ }^{18} \mathrm{Se}$ trataba, pues, de la figura convencional del dirigente político, sin otras competencias para la administración del área de la salud que la que le otorgaba el ejercicio de la medicina.

De manera que la designación de Carlos A. Bocalandro en el ministerio iba a suponer un cambio de rumbo. Se trataba de un médico del Cuerpo Sanitario del Ejército, de ahí, presuntamente, su vinculación con el gobernador. Era teniente coronel de Sanidad, grado inmediato al superior dentro del escalafón de sanidad. Su trayectoria había pasado por la Junta Superior de Reconocimientos Médicos, como cirujano de Brigada, y posteriormente por el Cuartel Maestre General del Interior, una dependencia encargada de abastecimiento y suministros del cuerpo con sede en la capital del país. Tenía pues experiencia en gestión y contralor, pero también, gracias a su contacto con los conscriptos, una visión de las necesidades sanitarias de la población. De hecho, con otros colegas del arma había realizado publicaciones sobre la incidencia de la tuberculosis y la nutrición entre los ingresantes al servicio militar en la Revista de la Sanidad Militar. Así, aunque no era médico sanitarista, su carrera lo distanciaba de los funcionarios que habían actuado hasta entonces en el área. ${ }^{19}$

El ministro era acompañado por dos subsecretarios, también médicos. Armando Falcone permaneció poco más de un año en la Subsecretaría de Salud Pública para continuar como comisionado en la ciudad de La Plata y más tarde como senador provincial. De manera análoga, Tito Pérez Otero, que le sucedió, se desempeñó en cargos partidarios que lo llevaron a la diputación nacional en 1952. La inserción en la lucha partidaria signó tanto su ingreso como la continuidad en la gestión. Distinta era la extracción del subsecretario de Acción Social, Fidel A. Maciel Crespo, que procedía del ámbito de la Universidad Nacional de La Plata, aunque tampoco completó su mandato. Poco sabemos de los tres funcionarios que le siguieron, aunque por su número inferimos la falta de estabilidad vigente. De manera que, en la medida que los directores y jefes de cada área dependían de las decisiones de los subsecretarios, sus recambios debieron afectar el quehacer del ministerio.

\footnotetext{
${ }_{18}^{17}$ Registro Oficial de la Provincia de Buenos Aires (RO), (1940-1952).

18 Citas históricas de Alberto $\quad$ O. Bítez https://www.saladillo.gob.ar/sites/default/files/alla_lejos_y_aqui_cerca.pdf

${ }^{19}$ Sobre la formación de los médicos del Ejército, ver Germán Soprano (2021). Los datos sobre este y otros funcionarios han sido obtenidos del cruce de diversas fuentes, entre otras: Boletín Oficial de la República Argentina, (17 de septiembre de 1942, p. 2); RO, (1940-1952); Quien es quien en la Argentina (1955); United States. War Department, (1947).
} 
Da Orden, M.L. Estado y salud pública en la provincia de Buenos Aires: proyectos y prácticas durante el peronismo clásico

Sin duda, esta situación debió reforzar el papel del ministro, que además tuvo una continuidad inusitada, ya que permaneció en su función durante las dos gestiones de Mercante (por la reforma constitucional de 1949, el gobernador fue reelecto al año siguiente para un mandato provisorio de dos años).

A mediados de 1952, con la llegada de Carlos Aloé a la gobernación, la cartera fue ocupada por Enrique Vattuone, un médico del que nada conocemos hasta 1944, cuando ocupó un cargo en la recién creada Secretaría de Salud Pública de la Nación. Como el propio gobernador y otros miembros del gabinete, provenía de la administración del gobierno central $y$, en consecuencia, propiciaba la aplicación indiscutida de sus lineamientos (Aelo, 2012). Es posible que los subsecretarios también tuvieran ese origen, aunque no hemos podido identificar su recorrido.

De este modo, si bien es cierto que todos los funcionarios del nuevo ministerio eran médicos, tanto por su trayectoria como por su dedicación a la gestión sanitaria conformaban un conjunto heterogéneo. En este sentido, más allá del cambio de signo político, su condición de profesionales de la salud abocados a la política poco los distanciaba de los funcionarios que tradicionalmente se habían desempeñado en el área. De ahí la importancia de una figura como la de Bocalandro en momentos en que el ministerio debía ponerse en marcha. En efecto, tanto los directores generales como los de división -algunos provenientes de secciones anteriores-y en general el personal que ahora formaba el gabinete debían adecuarse a los nuevos circuitos jerárquicos y administrativos y a los también nuevos alcances del área. Tenía que generarse así una tradición sobre la base de conocimientos e inercias preexistentes, una necesidad que, si bien excede el objetivo de este trabajo, no debería darse por sentada. ${ }^{20}$

Entre otras, las funciones del ministerio conllevaban la supervisión de los nuevos establecimientos de salud, desde su proyección junto con el Ministerio de Obras Públicas, hasta su habilitación. Ahora bien, ¿en qué medida se concretaron los planes de obras? La información fragmentaria de las fuentes hace difícil precisar la respuesta. No obstante, el detalle que ofrecen los presupuestos provinciales hasta 1950 permite una aproximación. A juzgar por las previsiones, para esa última fecha los servicios de salud habían aumentado significativamente. Además de la ampliación de los policlínicos de mayor complejidad -en La Plata, Haedo y Bahía Blanca-, los hospitales habían pasado de ser 15 a 27. Es cierto que tenían características muy dispares; de hecho, seis de ellos solo contaban con dos médicos y tres auxiliares, poco más que las denominadas unidades sanitarias. Estas -unas 40- también tenían capacidad de internación, así como 15 de las 91 salas de primeros auxilios y puestos sanitarios. ${ }^{21}$

Asimismo, parte de las obras destinadas a la asistencia social se ocupaban del tratamiento y prevención de "enfermedades sociales" ${ }^{22}$ y tuberculosis. Al respecto, se

\footnotetext{
${ }^{20}$ Más allá del tipo ideal de burocracia, seguimos aquí el enfoque propuesto por Ernesto Bohoslavsky y Germán Soprano (2010).

${ }^{21}$ DSCD, (1946-47, Tomo V, pp. 4208-4291); Texto del cálculo de recursos y presupuesto general de gastos para 1950. DSCD, (1949-50, Tomo IV, pp. 2893-2970). En 1952 se incorporaron otros seis hospitales (Ley 5671), entre ellos, el Instituto San Martín del Policlínico de La Plata, con 400 camas (Longoni, Galcerán y Molteni, 2007).

22 Entre otras, una enfermedad del ámbito rural como la hidatidosis fue puesta por primera vez en la agenda de salud pública (Valobra, 2007).
} 
Da Orden, M.L. Estado y salud pública en la provincia de Buenos Aires: proyectos y prácticas durante el peronismo clásico

sumaron algo más de cincuenta centros que permitían desconcentrar la tradicional atención en los anexos de los principales hospitales. No obstante, por el número de establecimientos, la prioridad correspondía a la atención del "binomio madre-hijo" (Valobra, 2005). Así, los centros de higiene materno-infantil aumentaron de 6 a 33, todos con camas para internación. Junto con los dispensarios y otros servicios -con médicos, obstétricas, enfermeras y visitadoras de higiene-, esta atención alcanzó, hacia 1950, a más de setenta partidos de la provincia. ${ }^{23}$

Con las limitaciones que supone todo diseño presupuestario, ${ }^{24}$ lo cierto es que la presencia sanitaria del poder provincial se había extendido a casi todos los municipios (103 de los 112 existentes), la tercera parte de los cuales -sin contar el de La Plata, principal beneficiario- fueron destinatarios de tres o más obras, entre refacciones, ampliaciones o construcciones nuevas. Se trataba de establecimientos de pequeña o mediana escala que brindaban atención primaria y de mediana complejidad en lugares donde el Estado provincial no se había hecho presente hasta entonces. ${ }^{25}$ Así, las 8742 camas existentes en 1951 casi duplicaban las que había tenido a su cargo la provincia un lustro antes. ${ }^{26}$

Si este resultado es impactante, lo cierto es que también reflejaba algunas limitaciones que escapaban al control del ministerio y aun al poder provincial. En efecto, el hecho de que la mayoría de las obras se hubieran inaugurado en los dos primeros años de gestión y que esa cifra representara un 30\% menos que la prevista da cuenta, entre otros aspectos, del impacto de la crisis económica (Gerchunof y Antúnez, 2002). En efecto, desde un principio, el aumento de los costos había sido un problema: ya a fines de 1946 se había autorizado el reajuste de las obras licitadas y la situación no iba a mejorar. Tres años más tarde, cuando la inflación trepó al 39\%, otras dos leyes aprobaron sendos aumentos con cargo al crédito público. Pese al Plan de Emergencia Económica implementado en 1952 por el gobierno nacional, dos años después, otra ley facultó al gobernador Aloé para que incrementara nada menos que hasta en un $40 \%$ los contratos vigentes. ${ }^{27}$ La falta de materiales y de mano de obra también tuvo consecuencias en el caso de las construcciones realizadas por el Ministerio de Obras Públicas. A los efectos de la coyuntura se sumaban aquí las demandas de Eva Perón, que llevaron a paralizar temporariamente las obras para derivar recursos hacia la FEP. ${ }^{28}$ Un hecho que da cuenta de los problemas que en la práctica suponía no solo la articulación de jurisdicciones sino también de los poderes existentes. Por lo demás, si esto afectaba al conjunto de las construcciones públicas, el encarecimiento o las

\footnotetext{
${ }^{23}$ MSPyAS, (1947-1951, p. 60); DSCD, (1949-50, Tomo IV, pp. 2974-3078).

${ }^{24}$ Así, por ejemplo, el hospital de Punta Alta, presupuestado para 1950, recién fue inaugurado tres años después. MSPyAS, (1947-1951, pp. 22-23); Ministerio de Salud Pública (MSP), (1952-1953), Memoria correspondiente al ejercicio del año.

${ }^{25}$ DSCD, (1949-50, Tomo IV, pp. 2948, 2954 y 2967).

${ }^{26}$ MSPyAS, (1947-1951, p. 76).

${ }^{27}$ Leyes 5070 de 1946, 5308 y 5427 de 1949. La medida de 1954 formaba parte de la normativa general de obras públicas (Ley 5806).

${ }^{28}$ Testimonio de Pedro Poggi, subsecretario obras públicas durante el gobierno de Mercante (Clarke, Ghisiglieri y Sarno, 2006).
} 
Da Orden, M.L. Estado y salud pública en la provincia de Buenos Aires: proyectos y prácticas durante el peronismo clásico

limitaciones a la importación incidían en la adquisición del equipamiento imprescindible para habilitar los establecimientos sanitarios. ${ }^{29}$

Como era de esperar dada la caída política de Mercante y su equipo de gobierno (Aelo, 2012), Vattuone, el ministro de salud de la nueva administración, responsabilizó a la gestión anterior por los problemas que atravesaba la cartera. No pudo dejar de mencionar, sin embargo, "la tiranía del régimen de economías" a la que debió adaptarse para excusar la labor "modesta, pero fecunda" del ministerio. ${ }^{30}$ Una labor que tampoco se modificó con la aprobación del Plan de Inversiones del Estado 1953/57, con el que la gobernación adhirió automáticamente al Segundo Plan Quinquenal implementado por la nación (Ley 5712). De esta suerte, solo pudieron inaugurarse obras que ya estaban a punto de ser concluidas y las memorias anuales se ciñeron a las estadísticas de controles y expedientes realizados.

Lo cierto es que, sea por los efectos de la crisis económica o por las dificultades que implicaba la gestión, el vasto plan de obras públicas del peronismo provincial alcanzó su tope hacia 1950. En los años sucesivos, el ministerio debió concentrar sus esfuerzos en mantener la estructura generada. Al menos en el ámbito de la salud pública y a pesar de sus diferencias políticas, la cuestión trascendía el marco de las gestiones de Mercante y Aloé. Si en las memorias del departamento el ministro Vattuone traducía la alineación incondicional con el gobierno nacional que caracterizó al nuevo gobernador -alineación que, por otra parte, también manifestara Bocalandro-, el hecho es que la restricción de los recursos provinciales constituía una razón suficiente para afectar la autonomía del área. En este sentido, más allá del avance del poder central que ha sido señalado para otros distritos (Bacolla, 2016; Ortiz Bergia, 2016), los efectos de la crisis y del manejo de las cuentas fiscales no deben desestimarse a la hora de considerar el margen de maniobra de sus políticas sanitarias (Hirschegger, 2016).

Con todo, a juzgar por el número de camas en relación con la población, la provincia de Buenos Aires se hallaba en mejores condiciones que Santa Fe o Córdoba, también embarcadas en importantes mejoras. En efecto, en 1951 el distrito contaba con 4,55 lechos cada 1000 habitantes, un indicador que en el mismo año era de 2,43 en la provincia del litoral y de 3,47 en la mediterránea para 1954 (Ortiz Bergia, 2015, Bacolla, 2016). ${ }^{31}$

La diferencia se incrementaba además por la acción del gobierno nacional y la FEP, que entre 1951 y 1954 habían construido los policlínicos de Avellaneda, Lanús y General San Martín, e incorporaban 1500 camas. $^{32}$ La importancia de la población obrera en estas localidades del Gran Buenos Aires, uno de los pilares del peronismo, no necesita subrayarse. De ahí que, a pesar de los planes y anuncios efectuados, el aporte del poder central en este caso no tuviera parangón con el realizado en otras provincias (Raffa, 2014; Bacolla, 2016). Esto acentuaba la brecha entre el distrito bonaerense y los restantes -el número de camas cada 1000 habitantes se elevaba así a 4,89-, pero lo

\footnotetext{
${ }^{29}$ Al respecto, en 1948 debieron reforzarse las previsiones que estipulaba el plan inicial (Ley 5378).

${ }^{30}$ MSP, (1952-1953, pp. 5-6; 7-12).

${ }^{31}$ Cálculo sobre la población del IV Censo General, (1951, Tomo 1).

32 La nación también había hecho construir un hospital para 1000 internos en Mar del Plata que, a pesar de haberse terminado en 1955, fue inaugurado seis años más tarde (Longoni et al., 2007).
} 
Da Orden, M.L. Estado y salud pública en la provincia de Buenos Aires: proyectos y prácticas durante el peronismo clásico

cierto es que los nuevos lechos gestionados por la provincia triplicaban los que había inaugurado la nación. En lo que este indicador se refiere, su protagonismo en la atención sanitaria de la población resultaba, pues, indiscutible.

Ahora bien, ¿con qué recursos contaba el ministerio para poner en marcha los nuevos establecimientos y las agencias que lo componían? Desde el punto de vista presupuestario, las partidas tuvieron un correlato con el impulso que buscó darse a la salud pública. En el año de creación, el $18 \%$ de los gastos del Poder Ejecutivo fue destinado a salud y asistencia social. Con el fuerte aumento que tuvo el presupuesto general, al año siguiente la proporción descendió al 12\% y tendió a estabilizarse en torno al 11\%, con la excepción de 1954, cuando el primer presupuesto de la gestión de Aloé lo aumentó en dos puntos. ${ }^{33}$ Se trataba de una asignación superior al 10\% propuesta por el gobierno nacional a las provincias (Belmartino et al., 1991), aunque algunas habían destinado un porcentaje mayor. ${ }^{34}$ Claro que, más allá de las previsiones, la cuestión clave es la efectiva ejecución de los gastos. Si consideramos que en un año tan significativo como 1952 para el rumbo económico (entre el gobierno de Mercante y Aloé), esos montos superaron en un $15,5 \%$ la partida prevista para el ministerio, mientras que las de toda la administración los habían aumentado un $10 \% .{ }^{35}$ Parece claro el apoyo que tenía la política sanitaria o, al menos, la presión que en su favor ejerció este departamento.

Sin embargo, tanto por el número de agentes como por la incorporación de nuevas cargas sociales, los mayores gastos estaban destinados al pago de sueldos. En efecto, entre 1947 y 1950 el personal aumentó en un 60\%, y llegó a 11.470 individuos en el último año. Las inauguraciones de 1952 incrementaron el número a 12.000 (Ley 5671), una cifra que no debió modificarse demasiado si tenemos en cuenta el freno que tuvo la obra pública y los ajustes a los que se refería el ministro Vattuone. Pese a ello, como nunca antes, la participación del área de Salud en el empleo público provincial del orden del $14 \%$ - solo era superada por Seguridad y Educación. ${ }^{36}$

Más allá del posible ingreso de "recomendados" de una u otra gestión, lo cierto es que la designación del personal sanitario planteó dificultades a veces insalvables para el ministerio. Por ejemplo, la tercera parte de los más de mil médicos destinados a los establecimientos provinciales debía tener una especialidad. Sin embargo, una de las cuestiones era hallar profesionales "a quienes convenga ausentarse a poblaciones del interior de la provincia", una realidad que tuvo consecuencias, en este caso, para la habilitación de cinco centros de salud mental. ${ }^{37}$ Pero además, cuando se conseguían, no siempre era posible retenerlos. Ese fue el caso del policlínico de La Plata, para el que se contrató un técnico en lugar de un médico anestesista, hecho que fue interpelado por un diputado de la oposición. La respuesta del ministro, presente en la Cámara, da

\footnotetext{
${ }^{33}$ Entre 1947 y 1948 el presupuesto general de la administración (sin el Poder Judicial) pasó de 231,7 a 478,4 millones de pesos respectivamente. Leyes 5245 y 5746; MSPyAS, (1947-19551, p. 21).

${ }^{34}$ El Ministerio de Salud de Córdoba siguió una tendencia inversa al bonaerense: pasó de una asignación presupuestaria del $8 \%$ a una del 16\% en 1951, para culminar con el 19\% en 1954 (Ortiz Bergia, 2016). La partida del área en Santa Fe -un promedio del 14\%-también fue algo mayor que la de Buenos Aires, en tanto que Mendoza siguió la tendencia de esta última (Bacolla, 2016; Hirschegger, 2016).

${ }^{35}$ Ministerio de Economía, Hacienda y Previsión, (1954). Memoria del ejercicio 1953.

${ }^{36}$ DSCD, (1946-47, Tomo V); (1949-50, Tomo IV).

${ }^{37}$ MSPyAS, (1947-1951, p. 47).
} 
Da Orden, M.L. Estado y salud pública en la provincia de Buenos Aires: proyectos y prácticas durante el peronismo clásico

cuenta de lo que decimos: "Para qué vamos a seguir hablando si sabemos que el único médico anestesista de La Plata es el doctor Aranes que se alejó de la ciudad de La Plata porque aquí no podía vivir con 500 pesos del presupuesto". Otro tanto señaló con respecto a distintos especialistas, desde cardiólogos y reumatólogos hasta endoscopistas y kinesiólogos: "le hago notar al señor Diputado [el radical Teófilo Naim] que en el Hospital de Niños de La Plata, para tener seis kinesiólogos, hube de dirigirme a la Facultad de Buenos Aires [sic]. Me enviaron seis nombres pero los kinesiólogos no vinieron". ${ }^{38} \mathrm{Si}$ el ministro manejaba con este detalle la información sobre el tema, parece evidente que la provisión de estos cargos no era un tema menor en el quehacer del ministerio.

Lo propio cabría señalar para el caso de las visitadoras o las enfermeras, cuyo número estaba prescripto de acuerdo con las características de las unidades sanitarias. Con vistas a las nuevas necesidades fue creada la Escuela de Auxiliares Técnicos bajo la órbita del departamento, que también aumentó las escuelas que funcionaban en los establecimientos sanitarios -35 entre 1949 y $1952-.{ }^{39}$ No obstante, los casi 1500 cargos previstos para 1950 representaban poco más de la mitad de los requeridos por los estándares de la Organización Panamericana de la Salud. ${ }^{40} \mathrm{Si}$ tiempo después, en los años sesenta, hubo un gran déficit en el área (Ramaciotti y Valobra, 2017), ¿cómo se cubrirían a fines de los cuarenta las demandas producidas en escasos tres años? No resulta sorprendente, entonces, que la partida para "Hermanas de Caridad" continuara figurando en el presupuesto provincial.

Todo ello da cuenta de la necesidad de considerar los distintos aspectos que pesaban en el día a día de la acción ministerial, y esto solo en relación con las instalaciones sanitarias. Necesariamente ello se traducía en el plano local. Veamos un caso concreto. Cuando se creó el departamento, el importante Policlínico de Bahía Blanca, ubicado en el sudoeste bonaerense, contaba con casi tres décadas de funcionamiento -había surgido en el marco de las políticas sanitarias del radicalismo provincial-. De ahí que el plan trienal lo incluyera entre los establecimientos que debían ampliarse y reformarse. Aunque en 1947 el presupuesto había duplicado el personal existente a comienzos de la década -143 cargos-, la mitad correspondía a tareas de servicio y mantenimiento. Los 22 profesionales asignados no solo debían cubrir las distintas especialidades del hospital, que tenía una capacidad de 300 camas, sino también los consultorios externos y esto en la jornada de tres horas que establecía la carrera médico-hospitalaria para la mayoría de ellos (Ley 5364). Más allá de la presencia de concurrentes (ad honorem), ello implicaba que la mayor parte del tiempo los internos debieran acudir al personal de enfermería. ${ }^{41}$ Sin embargo, la dotación de 48 puestos suponía que cada auxiliar debería atender a unos 15 pacientes -si en el turno

\footnotetext{
${ }^{38}$ DSCD, (1949-50, Tomo IV, pp. $\left.2694-2695\right)$.

${ }^{39}$ MSPyAS, (1947-1951, p. 49); MSP, (1952-1953, p. 177).

${ }^{40}$ En 1960 dicha organización estableció que se necesitaban 6 enfermeras y 27 auxiliares por cada 100 camas. Según esto, los establecimientos de la provincia deberían haber contado con 492 enfermeras y 2214 auxiliares de enfermería, distinción que a comienzos de los años cincuenta todavía no se realizaba, pero que da cuenta del problema (Ramaciotti y Valobra, 2017).

${ }^{41}$ Como señala Alberto Dal Bó (2008), si no hubiera sido por los médicos concurrentes, la mitad del día los hospitales habrían quedado desiertos.
} 
Da Orden, M.L. Estado y salud pública en la provincia de Buenos Aires: proyectos y prácticas durante el peronismo clásico

noche el personal se reducía a la mitad y no se producían inasistencias-. ${ }^{42}$ Una situación que no varió demasiado en 1950, cuando volvieron a sumarse cargos, si tenemos en cuenta la ampliación que había supuesto el pabellón de psiquiatría. Así, además de la disponibilidad de camas, el tipo de atención dependía mucho del movimiento de las internaciones.

De este modo, aunque el aumento de personal y las mejoras edilicias introdujeron cambios significativos, no resultaba fácil erradicar los problemas que implicaba la "cuestión hospitalaria". Cierto es que, a pesar de las transformaciones de la medicina, buena parte de la cura continuaba basándose en la internación, lo cual podría explicar la asignación y dedicación de los profesionales. En el mismo sentido debieron actuar las asociaciones médicas, celosas de la amenaza que podía suponer el hospital público para la consulta privada (Belmartino et al., 1991; Dal Bó, 2010). Estas y otras circunstancias condicionaban las prácticas del ministerio y sin duda estaban en la base del apoyo que recibían las organizaciones intermedias orientadas a la salud pública.

En efecto, aunque el Estado nacional había desplazado a la Sociedad de Beneficencia de Buenos Aires (Ramaciotti, 2009) y algunas provincias habían tomado medidas análogas con entidades locales (Hirschegger, 2016; Ortiz Bergia, 2016), ese no había sido el caso del distrito bonaerense. El propio ministerio tenía una partida para subsidiar tanto establecimientos municipales y vecinales como otros de carácter benéfico o confesional. Asimismo, además de las ampliaciones y refacciones que tomó a su cargo, que supusieron el aumento del número de camas de dichas entidades, la repartición estaba autorizada a donarles equipamiento -tal fue el caso de la mayoría de las ambulancias y equipos radiográficos adquiridos en 1949-. ${ }^{43}$ Del mismo modo, como se venía haciendo desde hacía décadas, el departamento de hacienda subsidiaba a más de dos centenares de hospitales y salas de primeros auxilios. ${ }^{44} Y$ es que la idea de coordinación de todas las instituciones que establecía la propia ley de creación del ministerio también daba cuenta de las limitaciones del gobierno provincial para hacerse cargo de la totalidad del sistema. En este sentido, la noción de coordinación interesa aquí como reconocimiento por el poder provincial de la fragmentación existente y, por ende, como continuidad dentro del cambio que supuso la creación del ministerio, un aspecto en el que, como vimos, coincidían oficialistas y opositores. Es este el punto de partida para considerar la configuración del sistema sanitario durante el peronismo antes que el ideal de unificación, por más deseable que fuera con vistas a la universalización de la salud.

Ahora bien, las limitaciones de la administración estatal -desde la inestabilidad de los altos funcionarios a la novedad de un departamento que debía funcionar a partir de agencias preexistentes al mismo tiempo que crecía aceleradamente-, habrían

\footnotetext{
42 De considerarse los estándares mencionados en la nota 40, la cifra se hubiera elevado a 99, casi el doble de lo asignado (Ramaciotti, Valobra, 2017).

${ }^{43}$ MSPyAS, (1947-1951, p. 94); Ley 5366.

${ }^{44}$ DSCD, (1949-50, Tomo IV, pp. 2809-2819). Nótese que el subsidio por cama que giraba ese departamento fue aumentado al mismo tiempo que se consideraba la creación del Ministerio de Salud (Ley 5050). Asimismo, en 1947 se dispusieron asignaciones para sueldos y gastos de cinco de hospitales sostenidos por sociedades de beneficencia que atravesaban serias dificultades financieras (Ley 5184).
} 
Da Orden, M.L. Estado y salud pública en la provincia de Buenos Aires: proyectos y prácticas durante el peronismo clásico

dejado un amplio margen para la acción personal. Aunque es un tema del que poco se conoce, algunos indicios sobre el estilo de gestión de Bocalandro apuntan en ese sentido. Sus visitas a distintas localidades decían buscar "la palpación y observación directa de los problemas" y su resolución "sobre el terreno". ${ }^{45}$ Independientemente de su intencionalidad política, ello indicaba lo que se consideraba necesario para el funcionamiento del área. Así, según el director del centro para el tratamiento de la tuberculosis de Tres Arroyos (localiza a 500 kilómetros al sur de la capital provincial), la conformidad del ministro con el estado del establecimiento había redundado en un intercambio cuyo interés amerita la extensión de la cita:

—Y Ud. Qué quiere, De Pierris? —No me animo a decírselo, doctor. Es mucha plata. — Diga, vamos a ver. Pida ahora porque tengo y no sé si el mes que viene podríamos. Una sala de cirugía torácica completa y un equipo Odelca para abreugrafías. Este aparato que Ud. vio en la sala de rayos es improvisado por nosotros, con un portátil y una cámara Leyca. - No pide nada, eh? Veo que no es manco para pedir. Bueno, está bien. Mándeme urgente una lista del instrumental que necesita, con tres presupuestos, aunque el conjunto no sea de una sola firma. Pero, eso sí, el material, después que yo le apruebe la compra, lo va a elegir Ud. personalmente, pieza por pieza, porque las casas proveedoras en estas licitaciones oficiales suelen meter al menor precio lo que tienen de rezago... Listas y presupuestos a mi casa de calle Malabia. El Odelca... voy a averiguar (mientras hacía anotar al secretario), porque me parece que en el depósito que tenemos en Melchor Romero tiene que haber tres, si ya entraron al país... Tuvimos la sala de cirugía torácica. Años después, mediante múltiples vicisitudes, nos hicimos del Odelca, al que le habían sacado el tubo. ${ }^{46}$

Más allá de la anécdota, el testimonio pone en evidencia distintos aspectos del quehacer del ministerio. Así, la articulación entre la administración central y el plano local dependía de la mayor o menor eficacia de los trabajadores de la administración, pero también de la disponibilidad efectiva de los recursos afectados (el "pida ahora porque tengo") o incluso del comportamiento de los licitantes. Aunque no es el caso de este ejemplo, otro tanto cabría decir de los auxiliares y especialistas de la medicina, cuya oferta y disponibilidad no debe darse por sentada. Se trata de aspectos específicos y diversos del funcionamiento que muestran la contracara de las normativas, organigramas o aun disposiciones presupuestarias. En la práctica y en la experiencia del ministro, el margen para la acción personal era bastante amplio y posiblemente necesario, al menos en un departamento que debía crear una tradición al mismo tiempo que ampliaba su esfera de actuación.

\footnotetext{
${ }^{45}$ MSPyAS, (1947-1951, p. 7).

${ }^{46}$ De Pierris, C. A. "Un Recuerdo...". Publicado en blog. Un testimonio análogo sobre el reclutamiento de un empleado administrativo del Policlínico de Bahía Blanca en: Testigos y protagonistas/Rafael Donnari. Cien años con el fuego en el alma (18 de abril de 2010). La Nueva. https://www.lanueva.com/nota/2010-4-18-90-0-cien-anos-con-el-fuego-en-el-alma
} 
Da Orden, M.L. Estado y salud pública en la provincia de Buenos Aires: proyectos y prácticas durante el peronismo clásico

\section{A modo de cierre}

Para realizar un balance algo más que provisorio, las cuestiones que aquí consideré deberían profundizarse. Nada dije, además, sobre las campañas de prevención y vacunación o de las acciones emprendidas frente a la periódica virulencia de algunas enfermedades y las prácticas de los sectores sociales a los que estaban destinadas. Con todo, puede afirmarse que la salud pública adquirió un nuevo rumbo con la llegada del peronismo al gobierno de la provincia de Buenos Aires. La extensión territorial y la variedad socioeconómica del distrito requerían de una política que aumentara la injerencia provincial en una realidad sanitaria que ya venía siendo objeto de críticas. El Ministerio de Salud Pública y Acción Social y los distintos planes de obras siguieron esa dirección.

Ahora bien, la puesta en marcha de la nueva cartera no puede darse por sentada. Así, si es cierto que los más altos funcionarios tenían la calificación que les daba su título de médicos y en algún caso una trayectoria académica, también lo es que su frecuente recambio -involucrados en la lucha partidaria como estaban- poco favorecía el funcionamiento de las distintas áreas. En particular cuando era necesario crear una dinámica ministerial -hacia adentro y hacia afuera-, con las inercias de las agencias preexistentes y la novedad de otras, al tiempo que se expandían como nunca antes. De ahí el margen abierto para la acción personal de la que da cuenta el ministro Bocalandro y posiblemente también de otros funcionarios y agentes.

Por lo demás, junto con la ampliación de los grandes hospitales, la concreción de una parte significativa de los planes de obras se hizo patente en los numerosos establecimientos de pequeña y mediana escala que llegaron a localidades donde el poder provincial no se había hecho presente hasta entonces. Los recursos y el personal asignado tampoco tenían precedentes. Sin embargo, a diferencia de otros distritos, esta intervención no buscó reemplazar la acción de los municipios ni de las instituciones civiles o confesionales. Las inversiones, subsidios y otros recursos evidencian el apoyo brindado por el gobierno provincial a las distintas entidades que funcionaban desde larga data. Aunque habría que considerar el contralor que esto suponía, esta relación no solo emergía de la práctica. En la concepción de los legisladores -oficialistas y opositores-, la unificación del sistema sanitario suponía la coordinación de las instituciones ya existentes antes que la absorción por el nuevo ministerio. Una política que, entre otras cosas, tenía en cuenta la limitación del gobierno provincial para hacerse cargo de la totalidad del sistema en el distrito.

A la fragmentación así asumida también contribuyó el gobierno nacional. A juzgar por el establecimiento de tres importantes hospitales en el conurbano, aquí la participación del poder central había sido más significativa que en otras provincias. Si esta y otras medidas implicaban una pérdida de autonomía para el gobierno bonaerense, lo cierto es que la crisis económica y el estado de las cuentas de la provincia no estuvieron al margen de los límites a que llegaron las políticas sanitarias hacia 1950, un hecho que fue más allá del cambio de gobernadores.

Así, la escala provincial ofrece un ángulo diferente para explicar los cambios y también las continuidades de la salud pública en el período. Las políticas nacionales, en particular la tan mencionada fragmentación, punto de partida de este trabajo, cobran 
Da Orden, M.L. Estado y salud pública en la provincia de Buenos Aires: proyectos y prácticas durante el peronismo clásico

aquí otra perspectiva. En efecto, ¿es posible mantener la unificación del sistema sanitario como parámetro de análisis cuando los dirigentes de una provincia como Buenos Aires concebían y gestionaban de otro modo el funcionamiento de la salud? A juzgar por este y otros casos, la configuración de la salud pública en la esfera del Estado nacional no se reduciría a las limitaciones que la FEP o el propio Perón impusieron a Ramón Carrillo y sus políticas sanitarias. La intervención muchas veces superpuesta de las distintas jurisdicciones -central, provincial y municipal- y la continuidad de las organizaciones sociales preexistentes constituyen otras tantas dimensiones a tener en cuenta a la hora de explicar la persistente fragmentación del sistema sanitario en la Argentina peronista.

\section{Referencias bibliográficas}

1. Aelo, O. (2012). El peronismo en la provincia de Buenos Aires (1946-1955). Editorial de la Universidad Nacional de Tres de Febrero.

2. Anuario Argentina Ilustrada. Year Book. (1953). River Plate Publishing Co.

3. Anuario Geográfico Argentino. (1941). Comité Nacional de Geografía.

4. Armus, D. y Belmartino S. (2001). Enfermedades, médicos y cultura higiénica. En A. Cattaruza (Dir.) Crisis económica, avance del Estado e incertidumbre política. Nueva Historia Argentina. Tomo 7 (pp. 283-329). Sudamericana.

5. Bacolla, N. C. (2016). Nuevas capacidades estatales para una sociedad transformada. Instituciones y políticas sanitarias en la provincia de Santa Fe primera mitad del siglo XX. Trabajos y comunicaciones, 44, 1-25. https://www.trabajosycomunicaciones.fahce.unlp.edu.ar/article/view/TyCe023

6. Belmartino, S. (2005). La atención médica argentina en el siglo XX. Instituciones y procesos. Siglo XXI.

7. Belmartino, S., Block, C., Carnino, M. y Persello, V. (1991). Fundamentos históricos de la construcción de relaciones de poder en el sector salud: Argentina 1940-1960. Organización Panamericana de la Salud, Oficina Regional de la Organización Mundial de la Salud, Representación de Argentina.

8. Biernat, C. (2016). Continuidades y rupturas en el proceso de centralización de la administración sanitaria argentina (1880-1945). Trabajos y Comunicaciones, 44, $1-23$.

https://www.trabajosycomunicaciones.fahce.unlp.edu.ar/article/view/TyCe021

9. Boletín Oficial de la República Argentina. (1942). https://archive.org/details/Boletin_Oficial_Republica_Argentina_1ra_seccion_194 2-09-17/page/n1?q=bocalandro 
Da Orden, M.L. Estado y salud pública en la provincia de Buenos Aires: proyectos y prácticas durante el peronismo

10. Bohoslavsky, E. y Soprano, G. (2010). Un Estado con rostro humano. Funcionarios e instituciones estatales en Argentina (de 1880 a la actualidad). Universidad Nacional de General Sarmiento/Prometeo.

11. Clarke, G., Ghisiglieri, J. y Sarno, A. (2006). La Gobernación de Mercante. Construcción histórica con documentos orales. En C. Panella (Comp.) El gobierno de Domingo A. Mercante en Buenos Aires (1946-1952). Un caso de peronismo provincial. Tomo 2 (pp. 191-236). Instituto Cultural de la Provincia de Buenos Aires.

12. Dal Bó, A. (2008). Hospitales de reforma. Crónicas para evitar el olvido. Biblos.

13. De La Vega, G. (2017, 9 de agosto). El rubro de "Economía" dentro del Primer Plan Quinquenal: su financiación y resultados globales de las obras públicas [ponencia]. XVI Jornadas Interescuelas/Departamentos de Historia. Mar del Plata,Argentina.https://interescuelasmardelplata.files.wordpress.com/2017/09/1 06-de-la-vega.doc

14. Diario de Sesiones. Cámara de Diputados de la Provincia de Buenos Aires. (1946-47). Tomos I, IV, V. Dirección de Impresiones Oficiales.

15. Diario de Sesiones. Cámara de Diputados de la Provincia de Buenos Aires. (1949-50). Tomo IV. Dirección de Impresiones Oficiales.

16. Diario de Sesiones. Cámara de Senadores de la Provincia de Buenos Aires. (1946-47). Tomo III. Dirección de Impresiones Oficiales.

17. Gerchunoff, P. y Antúnez, D. (2002). De la bonanza peronista a la crisis de desarrollo. En J. C. Torre (Dir.) Los años peronistas (1943-1955). Nueva Historia de la Nación Argentina. Tomo 8 (pp. 125-205). Sudamericana.

18. Hirschegger, I. (2016). La Salud Pública frente a un Estado centralizado: establecimientos y servicios asistenciales en la provincia de Mendoza durante el primer peronismo. Trabajos y Comunicaciones, 44, 1-25. https://www.trabajosycomunicaciones.fahce.unlp.edu.ar/article/view/TyCe026

19. Longoni, R., Galcerán, V. y Molteni J. (2007). La infraestructura para la Salud Pública en la Provincia de Buenos Aires (1946-1952). En C. Panella (Comp.) El gobierno de Domingo Mercante: un caso de peronismo provincial. Tomo III (pp. 77-106). Instituto Cultural de la Provincia de Buenos Aires.

20. Ministerio de Asuntos Técnicos. Presidencia de la Nación. (1951). IV Censo General de la Nación. Tomo 1. Censo de Población. Dirección Nacional del Servicio Estadístico. 
Da Orden, M.L. Estado y salud pública en la provincia de Buenos Aires: proyectos y prácticas durante el peronismo clásico

21. Ministerio de Economía, Hacienda y Previsión. Provincia de Buenos Aires. (1954). Memoria del ejercicio 1953.

22. Ministerio de Obras Públicas. Provincia de Buenos Aires (1947). Plan General de Trabajos Públicos para el trienio 1947, 1948 y 1949. Volumen 1. Taller de Impresiones Oficiales.

23. Ministerio de Salud Pública y Asistencia Social. Provincia de Buenos Aires. (1947-1951). Memoria. Dirección de Prensa y Publicaciones.

24. Ministerio de Salud Pública. Provincia de Buenos Aires (1952-1953). Memoria correspondiente al ejercicio del año. Dirección de Impresiones Oficiales.

25. Ortiz Bergia, M. J. (2015) La temprana descentralización de los servicios de salud en la Argentina: la construcción del sistema sanitario en Córdoba, 1930-1955. História, Ciências, Saúde-Manguinhos, 22 (2), 559-575. https://doi.org/10.1590/S0104-59702015005000005

26. Ortiz Bergia, M. J. (2016). La centralización estatal en la Argentina y el sistema de salud público cordobés en la primera mitad del siglo XX. Trabajos $y$ comunicaciones, $\quad 44,416$. https://www.trabajosycomunicaciones.fahce.unlp.edu.ar/article/view/TyCe022

27. Quien es quien en la Argentina. Biografías contemporáneas. (1955). Kraft. https://archive.org/details/quienesquienenl00quiegoog

28. Raffa, C. (2014, 18 de septiembre). Lo dicho y lo hecho. Ideas, proyectos y concreciones arquitectónicas de la Fundación Eva Perón en Mendoza (19481955) [ponencia]. IV Congreso de Estudios sobre el Peronismo, Red de Estudios sobre el Peronismo. Tucumán, Argentina. http://redesperonismo.org/articulo/lodicho-y-lo-hecho-ideas-proyectos-y-concreciones-arquitectonicas-delafundacion-eva-peron-en-mendoza-1948-1955/

29. Ramaciotti, K. (2009). La política sanitaria del peronismo. Biblos.

30. Ramaciotti, K. y Valobra, A. (2017). El dilema Nightingale: controversias sobre la profesionalización de la enfermería en Argentina 1949-1967. Dynamis, 37 (2), 367-387.

31. Registro Oficial de la Provincia de Buenos Aires. (1940-1952). Taller de Impresiones Oficiales. 
Da Orden, M.L. Estado y salud pública en la provincia de Buenos Aires: proyectos y prácticas durante el peronismo clásico

32. Rodríguez, M. L. (2013). Las políticas hospitalarias peronistas: el caso de la provincia de Córdoba, Argentina (1946-1955). HiSTOReLo, 5 (9), 283-317. https://doi.org/10.15446/historelo.v5n9.34369

33. Ross, P. (2007). State Planning and the Public Health Sector in Argentina 19461955: Successes, Failures and Contradictions. Journal of Iberian and Latin American Studies, 13 (2), 29-53.

34. Senado de la Provincia de Buenos Aires. (1946-47). Diario de sesiones. Tomo III. Dirección de Impresiones Oficiales.

35. Soprano, G. (2021). Formación y perfil profesional de los médicos del Ejército Argentino a principios del siglo XX. Trabajos y Comunicaciones, 53, 1-18. http://dx.doi.org/10.24215/23468971e133

36. United States. War Department. (1947). Technical Bulletin Medical and Sanitary Data on Argentina. Government Printing Office. https://play.google.com/books/reader?id=nJ1YAAAAMAAJ\&hl=es_419\&pg=GB S.PP1

37. Valobra, A. (2005). De cronopios y de famas: la atención del binomio madre-hijo en la política sanitaria bonaerense durante la gobernación de Domingo Alfredo Mercante, 1946 y 1952. En C. Panella (Comp.) El gobierno de Domingo Mercante: un caso de peronismo provincial (pp. 129-172). Archivo Histórico de la Provincia de Buenos Aires.

38. Valobra, A. (2007). Un desafío a la justicia social peronista: la hidatidosis en la provincia de Buenos Aires, 1946-1952. História, Ciências, Saúde-Manguinhos, 14 (4), 1357-1375. http://dx.doi.org/10.1590/S0104-59702007000400014

39. Veronelli, J. C. y Veronelli Correch, M. (2004). Los orígenes institucionales de la Salud Pública en la Argentina. Tomo 2. Organización Panamericana de la Salud. 